
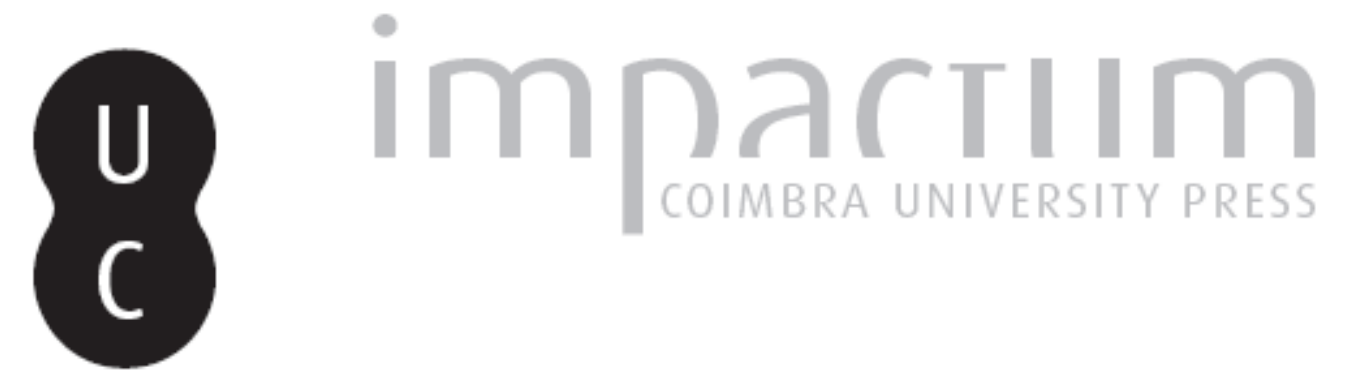

\title{
Plaisir et amitié: Homère et Aristote en dialogue
}

Autor(es): Collobert, Catherine

Publicado por: Sociedade Brasileira de Estudos Clássicos

URL persistente:

URI:http://hdl.handle.net/10316.2/35764

DOI:

DOI:http://dx.doi.org/10.14195/2176-6436_22-1_1

Accessed : $\quad$ 26-Apr-2023 00:43:07

A navegação consulta e descarregamento dos títulos inseridos nas Bibliotecas Digitais UC Digitalis, UC Pombalina e UC Impactum, pressupõem a aceitação plena e sem reservas dos Termos e Condições de Uso destas Bibliotecas Digitais, disponíveis em https://digitalis.uc.pt/pt-pt/termos.

Conforme exposto nos referidos Termos e Condições de Uso, o descarregamento de títulos de acesso restrito requer uma licença válida de autorização devendo o utilizador aceder ao(s) documento(s) a partir de um endereço de IP da instituição detentora da supramencionada licença.

Ao utilizador é apenas permitido o descarregamento para uso pessoal, pelo que o emprego do(s) título(s) descarregado(s) para outro fim, designadamente comercial, carece de autorização do respetivo autor ou editor da obra.

Na medida em que todas as obras da UC Digitalis se encontram protegidas pelo Código do Direito de Autor e Direitos Conexos e demais legislação aplicável, toda a cópia, parcial ou total, deste documento, nos casos em que é legalmente admitida, deverá conter ou fazer-se acompanhar por este aviso.

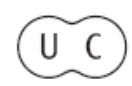




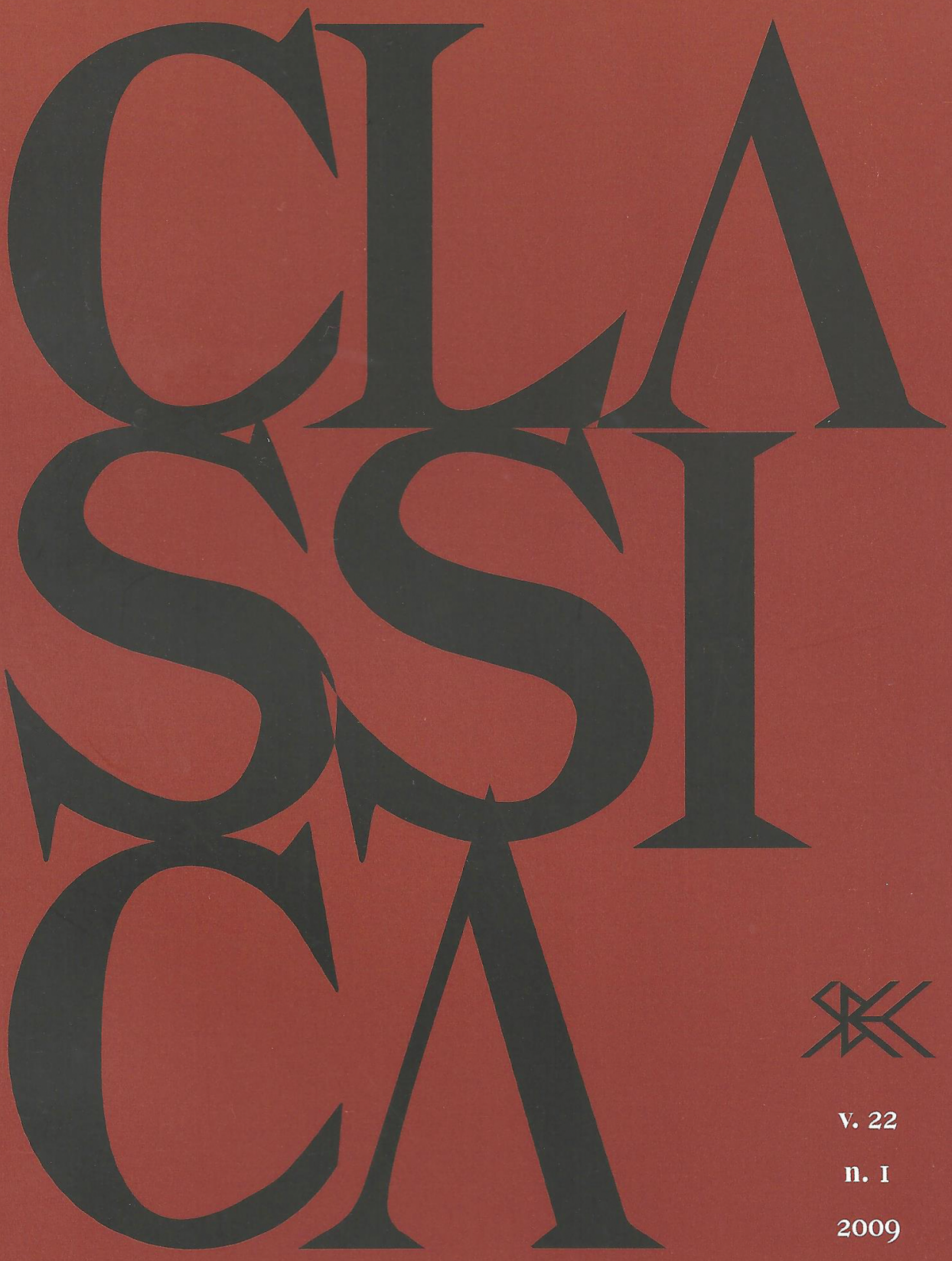




\title{
Plaisir et amitié: Homère et Aristote en dialogue
}

\author{
Catherine Collobert \\ University of Ottawa \\ Canadá
}

RÉsumé. L'affection et la compassion ont en commun d'être des emotions sociales, mais si la première est accompagnée de plaisir, la seconde est accompagnée de peine, selon Aristote. Il est cependant possible, selon Homère à la différence d'Aristote, d'éprouver de la compassion pour l'ami. Nous interrogeons ici le lien entre compassion et affection et montrons qu'en tant qu'émotions, elles sont constituées d'un désir d'agir en vue du bien de l'autre, c'est-à-dire d'un désir altruiste chez Homère. La satisfaction de ce désir produit un plaisir double qui prend la forme du contentement et de l'apaisement. Nous illustrons notre propos en établissant un dialogue entre Homère et Aristote.

Mots-CLÉs. Amitié; affection; compassion; plaisir; apaisement; contentement; émotion; intimité; obligation; désir; altruisme; vulnérabilité; Aristote; Homère.

Il est souvent affirmé que l'amitié en Grèce ancienne se distingue de l'amitié en son sens moderne. La première se caractérise par l'absence du caractère intime de la relation, par un lien d'obligations réciproques, de devoirs de l'un à l'égard de l'autre, qui fait de l'amitié un lien très légalisé et formel' ${ }^{1}$. Nous allons voir que l'intimité et l'obligation réciproque d'une part ne sont pas mutuellement exclusives, et d'autre part, que l'amitié implique la réciprocité en un sens très spécifique. C'est en tant qu'émotions altruistes actives qu'il faut comprendre l'articulation de ces deux émotions que sont l'affection et la compassion, laquelle articulation dessine les contours de l'amitié chez Homère.

Je défendrai la thèse qu'il existe entre l'affection et la compassion une relation d'implication qui définit la compassion comme constitutive

Email: ccollobe@uottawa.ca

${ }^{1}$ Cf. E. Benveniste, Le vocabulaire des institutions indo-européennes, vol. 1, Paris, Minuit, 1969, pp. 335-353, A. W. H. AdKIns, Merit and Responsibility : A Study In Greek Values, Oxford, Clarendon Press, 1970. Il faut noter que l'un n'exclut pas l'autre. Il y a un usage institutionnel du terme philos qui coexiste avec le premier sens de philos en tant que dénotant le lien d'affection entre deux personnes, comme l'affirme J. Hooker, Homeric qí̉os, Glotta 65, 1987, p. 63. 
de l'amitié. Je distinguerai dans un premier temps les facteurs principaux de la compassion et de l'affection. Nous verrons dans un second temps que le désir altruiste, c'est-à-dire le désir d'agir en vue du bien d'autrui, est ce qui forge le lien entre ces deux émotions. Dans un troisième temps, j'interrogerai le plaisir et la peine qui accompagnent ces émotions et montrerai ensuite que la satisfaction du désir altruiste produit dans le cas de ces deux émotions, compassion et affection, cette forme de plaisir qu'est la réplétion ou apaisement, et l'amitié cette forme de plaisir achevé qu'est le contentement ou la joie. Je conclurai sur la dimension éthique de l'amitié en montrant comment elle constitue un obstacle à des émotions et attitudes moralement condamnables. Mon propos se présentera sous la forme d'un dialogue entre Homère et Aristote, c'est-à-dire d'une confrontation des textes de la Rhétorique et de l'Éthique à Nicomaque avec ceux de l'épopée homérique.

\section{Les trois facteurs principaux de la compassion et de l'affection}

J'examinerai d'abord les facteurs principaux de la compassion et de l'affection en tant qu'attitudes évaluatives ${ }^{2}$ pour montrer comment l'amitié peut être comprise chez Homère comme une synthèse de ces deux émotions. Il me faut toutefois préalablement préciser la distinction entre pitié et compassion et les raisons pour lesquelles il est préférable d'utiliser le terme compassion. Comprise dans son acception chrétienne (la pitié de Dieu pour les hommes), l'émotion de pitié sous-tend l'idée d'inégalité, d'où la possibilité de la part de celui qui est objet de pitié d'un sentiment d'insulte voire d'humiliation ${ }^{3}$. En outre, la pitié implique l'absence du désir d'agir pour aider l'autre parce que le fait de nous sentir impuissants face à la souffrance de l'autre est, en partie, constitutif de l'émotion. Elle suppose, par conséquent, une relative acceptation de la situation. À l'inverse, la compassion implique un désir d'aider l'autre et de se sentir capable de le faire. Et enfin, motivée par la crainte, la pitié possède un aspect égoïste. Nous allons voir qu'il entre dans la pitié grecque une certaine forme de compassion et que, par conséquent, la distinction tranchée entre ces deux émotions par de nombreux théoriciens contemporains n'est pas pertinente en Grèce ancienne.

\footnotetext{
${ }^{2}$ Elle implique, selon les termes d'un théoricien contemporain des émotions A. Ben Ze'ev, «une position négative ou positive à l'égard de l'objet» (A. BEN ZE'Ev, The Subtlety of Emotions, Cambridge, MIT Press, 2000, p. 61, ma traduction).

${ }^{3}$ Cf. A. Ben ZE'ev, The Subtlety..., p. 329.
} 
Les trois facteurs principaux nécessaires pour soulever l'affection et la compassion sont relatifs à (1) l'objet de l'émotion (dans le cas de l'affection : le caractère de l'ami, dans le cas de la compassion : la situation malheureuse, les causes de cette situation); (2) le sujet à l'émotion (dans le cas de l'affection : le désir d'association, dans le cas de la compassion : le sentiment de vulnérabilité); (3) la relation entre le sujet et l'objet de l'émotion. L'affection est comprise chez Aristote comme un acte (to philein) : aimer, traiter avec affection et se distingue de l'amitié (substantif), qui est une relation spécifique (EN 1157b29 : l'affection (philêsis) est une émotion, l'amitié (philia) une disposition $)^{4}$. L'affection est considérée comme universelle, mais toute chose n'est pas aimable, c'est-à-dire ne suscite pas une réaction. Les choses aimables susceptibles de provoquer l'émotion d'affection sont celles qui sont bonnes, plaisantes ou utiles (1155b 18-20). Le bon, le plaisant et l'utile sont ainsi considérés comme les trois motifs de l'émotion. Émotion miroir, l'affection implique la réciprocité puisqu'elle est suscitée par l'intention de

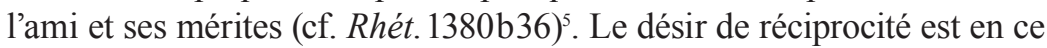
sens fondé sur l'affection. La réciprocité est fondamentale (Rhét. 1155b27) parce que l'affection qui ne serait pas payée en retour serait source de frustration et soulèverait des émotions négatives comme la colère et le ressentiment. L'affection mutuelle d'une part produit une mutuelle bienveillance (eunoia, cf. EN 1155b21) dont l'assistance est une preuve et une manifestation, et d'autre part, elle est le résultat d'une mutuelle sympathie ${ }^{6}$. Nous sommes réciproquement bien disposés l'un à l'égard de l'autre. L'affection s'exprime dans une attitude favorable à l'égard de l'ami : «En aimant leur ami, ils aiment leur propre bien» (EN 1157b33-35).

J'en viens maintenant à la compassion. Celle-ci est ressentie au spectacle d'un mal immérité cause de souffrances. L'émotion implique ici un jugement positif sur la personne (elle ne l'a pas mérité) et négatif sur ce qu'elle subit. Nous avons ici deux types d'évaluation l'une positive, l'autre négative : le mal est clairement distingué de celui qui le subit. Celui qui suscite la compassion n'est pas responsable de sa situation, s'il l'était il ne

${ }^{4}$ Le verbe est également employé chez Homère pour traduire l'affection des dieux pour les hommes et des hommes entre eux (cf. H. KaKRIDIs, La notion de l'amitié et de l'hospitalité chez Homère, Thèse, Thessaloniki, 1963, p. 40).

${ }^{5}$ Cf. D. Konstan, The Emotions of the Ancient Greeks. Studies in Aristotle and Classical Literature, Toronto, University of Toronto Press, p. 177. L'affection et l'amitié sont parfois très difficilement distinguables chez Homère, comme le remarque H. Kakridis (La notion ..., p. 22). ${ }^{6}$ L'amitié est, comme l'affirme D. Lefebvre «une bienveillance réciproque et déclarée fondée sur le caractère vertueux que chacun des amis semblables aime en l'autre» (D. Lefebvre, 'Bonheur et amitié', Aristote, Bonheur et vertu, Paris, Vrin, p. 161). 
serait pas objet de compassion? ${ }^{7}$ Le caractère immérité du mal (sans raison) est ici crucial. Celui en outre qui se met dans une situation désastreuse par manque de prudence n'inspire pas la compassion. Achille rejette la possibilité de la compassion pour les Grecs car ils périssent par leur faute. Celui qui a acquis sa fortune malhonnêtement et se trouve ruiné par un coup de la fortune n'inspire pas de compassion, mais, au contraire, un contentement qui implique le jugement «il a mérité son sort». Le jugement de contentement est ici l'opposé du jugement de compassion. Si donc l'agent est responsable de son malheur, il est vraisemblable, selon Aristote, que nous, qui sommes gens de bien et prudents, ne serons pas atteints par un malheur similaire.

Deuxièmement, nous sommes sujets à la compassion parce que nous savons qu'il peut nous arriver quelque chose de similaire. La condition psychologique de celui qui éprouve l'émotion est la conscience de sa vulnérabilité et de sa fragilité : nous pourrions à notre tour subir ce que l'autre subit. Mais il s'agit d'une vulnérabilité à la fortune, non à la faute morale ou à un manque de prudence. La conscience de la vulnérabilité doit cependant suscitée une émotion spécifique pour produire la compassion. En ce sens, la compassion n'est pas une émotion pure, mais s'accompagne d'une autre émotion qui entre dans sa production, qui est la peur, comme l'observe Aristote. Il s'agit d'une peur prospective qui exige une proximité temporelle. La peur requiert une forme d'immédiateté qui est transmise à la compassion. Ce lien essentiel avec la peur pour soi ou pour ses proches fait de la compassion une émotion à la fois tournée vers l'autre et tournée vers soi.

Cela me conduit au troisième facteur. Celui vers qui est dirigé la compassion est en situation d'infériorité, c'est pourquoi nous pouvons l'aider, mais il ne nous est pas inférieur. La crainte qu'un mal similaire nous arrive nécessite qu'il soit notre semblable. La tragédie soulève chez l'auditoire la compassion pour un héros tragique loin de nous être inférieur. Il y a une dialectique subtile entre proximité et distance dans le type de relation qui nous occupe ici. La compassion suppose une forme d'identification avec l'autre, mais aussi une certaine distance, qui maintient l'autre dans son altérité - la compassion se distingue en cela de l'empathie ${ }^{8}$. La distance

\footnotetext{
${ }^{7}$ Dans une oraison, Lysias affirme qu'il ne faut pas avoir pitié de ceux qui commettent des injustices, mais plutôt éprouver pour eux de la colère (15.9), Aristote dirait de l'indignation (puisqu'elle est, selon lui, l'opposée de la pitié. Elle consiste à ressentir de la peine pour quelqu'un qui réussit de manière imméritée).

${ }^{8}$ Comme le note D. Konstan, «feeling the same pain or pleasure as another does not mean experiencing the same emotion, since pain and pleasure are not themselves pathê. If my son is afraid of a monster, I may share something of his anguish, but I do not share his
} 
est nécessaire d'une part, pour que la peur ne devienne pas l'émotion dominante rendant impossible la production de la compassion, transformant le pitoyable en terrifiant (SEıvòv) (cf. Rhét.1386a20-24), et d'autre part, pour pouvoir agir, c'est-à-dire soulager l'autre de ses souffrances. La distance est la condition pour que l'émotion ne soit pas paralysante. Bien que l'identification consiste à faire de l'autre notre semblable, il y a plusieurs manières d'être semblables, c'est-à-dire aussi de s'identifier à l'autre.

La clé de l'identification est dans le cas de la compassion la vulnérabilité : on est semblable à l'autre du fait d'avoir en partage la vulnérabilité. C'est pourquoi la compassion devient possible entre ennemis, comme le montrent Homère et, après lui, Sophocle. Elle est possible entre ennemis, parce que comme l'affirme Achille, il est semblable à ce troyen : un guerrier qui périra sous les coups d'un autre, ce qui le conduit à l'épargner. De même Ulysse compatit à la folie d'Ajax, bien qu'étant un ennemi, préciset-il, parce qu'il reconnaît sa propre vulnérabilité (Ajax 118-32).

Cependant, pour ressentir de la compassion, il n'est pas nécessaire que l'autre soit dans une situation qui exprime une vulnérabilité dont nous pourrions faire l'expérience. En d'autres termes, l'expression de la vulnérabilité à travers une situation spécifique nous reconduit à notre propre vulnérabilité. Il est possible d'éprouver de la compassion pour une amie cardiaque bien que n'ayant aucun risque de contracter la maladie. Mais sa maladie nous renvoie à notre vulnérabilité à la maladie. Il y a identification parce que nous avons la possibilité de généraliser une expérience douloureuse, par conséquent, de la faire nôtre en un sens restreint. Nous pouvons donc compatir aux souffrances d'autrui sans craindre d'endurer quelque chose de semblable. La crainte dont il s'agit ici est une émotion dont l'objet est la vulnérabilité en son sens le plus général et qui nous incline à partager les maux et les souffrances d'autrui'. C'est l'expérience de la souffrance de la perte de l'ami, c'est-à-dire la vulnérabilité à la perte ${ }^{10}$, qui rend Achille compatissant aux souffrances de Priam.

Notons à cet égard que les mots de la famille oiktos qui expriment la compassion chez Homère (oiktirein: avoir pitié, oiktros : pitoyable, oiktistos, superlatif) sont associés à des titres divers à des figures de la vulnérabilité. Oiktos est ainsi parfois associé à l'humiliation : on ressent

fear, because I do not believe in monsters» (The Emotions..., p. 214).

${ }^{9}$ Comme le reconnaît H. Bergson «Peut-être la crainte entre-t-elle en effet pour quelque chose encore dans la compassion que les maux d'autrui nous inspirent» (Essai sur les données immédiates de la conscience, Paris, PUF, 1989, p.27).

${ }^{10} \mathrm{Cf}$. D. Hammer, The Iliad as Ethical Thinking : Politics, Pity, and the Operation of Esteem, Arethusa 35, 2002, p. 221. 
de la compassion pour quelqu'un qui se trouve dans une situation d'humiliation qui s'accompagne de souffrance. Ainsi Télémaque saisi par la colère et les larmes après son réquisitoire contre les prétendants soulève la compassion dans le peuple (Od.2.80-3). Celle-ci joue ici un rôle inhibiteur (le peuple se tient silencieux n'osant dire des mots cruels) ${ }^{11}$. Sur les cinq occurrences d'oiktros et sur les sept de la forme superlative, nous avons une association avec la mort ${ }^{12}$.

Il y a dans la compassion un détachement devant la particularité de la souffrance, qui en fait une émotion altruiste. Ce détachement rend les êtres humains susceptibles d'être l'objet de la compassion des dieux. Les êtres humains leur sont proches et lointains. Ils ne leur sont pas suffisamment lointains pour que les dieux se désintéressent de ce que les êtres humains font, pas davantage pour qu'ils ne puissent pas s'identifier à eux. La distance entre êtres humains et dieux s'exprime toutefois dans une relation d'infériorité : les dieux d'Homère ressentent de la compassion pour les êtres humains qui leur sont inférieurs. Les dieux sont en effet supérieurs aux humains par leur puissance, leur immortalité, leur absence de vieillesse, autrement dit, par une certaine forme d'invulnérabilité. Pourtant, ils n'en sont pas moins soumis aux émotions : ils connaissent la peur, celle d'Apollon face à Poséidon, la colère, celle d'Héra et d'Athéna pour les Troyens ou d'Apollon pour Agamemnon. Ils ont une vie émotionnelle aussi riche et variée que celle des êtres humains. Or l'émotion est le signe d'une vulnérabilité puisqu'elle est essentiellement un trouble, une déstabilisation du fait d'une situation non parfaitement maîtrisée et contrôlée ${ }^{13}$. Les dieux partagent donc avec les hommes une certaine forme de vulnérabilité et sont en cela leurs semblables (Zeus est père des hommes et des dieux). La compassion des dieux pour les hommes est exprimée par les mots de la famille d'éléos (eleein, eleairein, eleeinos dans sa forme adjectivale) ${ }^{14}$.

Les dieux sont compatissants lorsque leurs protégés se trouvent dans la bataille en situation d'infériorité et que la menace de la mort pèse sur

${ }^{11}$ Cf. M. Scotт, Pity and Pathos in Homer, Acta Classica 22, 1979, p. 7. La compassion d'Achille est exprimée à l'aide du verbe oiktirein à une exception près ioktirôn: Il. 24.516). ${ }^{12}$ Quatre occurrences dans l'Odyssée : 4.716-9; 10.408-9; 19.541-3 ; 24.58-59; une dans l'Iliade : 11.241-4. Pour la forme superlative : Od. $11.412 ; 12.258 ; 12.341-2 ; 22.472$; 23.79 ; 24.34 ; Il. 22.71-6.

${ }^{13}$ Cf. M. Nussbaum qui affirme que l'émotion en général «records that sense of vulnerability and imperfect control» (Upheavals of Thought : The Intelligence of Emotions, Cambridge, Cambridge University Press, 2003, p. 43).

${ }^{14}$ Pour une analyse du terme et ses différentes occurrences dans l'épopée, voyez J. KIM, The Pity of Achilles. Oral Style and the Unity of the Iliad, Lanham, Rowman \& Littlefield Publishers, Inc., 2000. 
eux $^{15}$. Comme l'affirme justement F. Nietzsche s'inspirant de Platon (Lois 906a4-5):

Les grecs ne voyaient pas les dieux homériques au-dessus d'eux comme des maîtres, et eux-mêmes au-dessous d'eux comme des valets, ainsi que les juifs. Ils ne voyaient en eux que le mirage des exemplaires les plus réussis de leur propre caste, c'est-à-dire un idéal, et non le contraire de leur propre être. On se sent parents les uns des autres, il se forme un intérêt réciproque, une espèce de symmachie... (Humain, trop humain, I, 114, trad. Desrousseaux \& Albert)

Ils sont, comme les humains, vulnérables à la souffrance, qui les rend capables de partager leurs souffrances. Zeus compatit aux souffrances d'Achille pour la mort de Patrocle, parce qu'il éprouve les mêmes souffrances qu'Achille pour la mort de Sarpédon. Il compatit aux souffrances d'Ulysse car bien qu'il ne puisse craindre de subir ses souffrances, il généralise à l'instar des humains la souffrance particulière d'Ulysse ${ }^{16}$.

\section{Le désir altruiste constitutif de l'affection et de la compassion}

L'émotion est constituée d'un désir de répondre à la situation présente et si besoin est de la modifier. La composante désir s'explique par l'appel à l'action et l'élan vers l'action qu'est l'émotion (readiness to act). Elle déclenche en même temps qu'elle prépare à l'action : elle est en ce sens une mobilisation des ressources ${ }^{17}$. Cependant, le désir est soit constitutif de l'émotion soit il suit l'émotion. La peur provoque le désir de fuite, mais le désir n'est pas constitutif de la peur, comme le souligne Aristote.

Nous allons voir que dans le cas de l'affection comme de la compassion, le désir altruiste est constitutif de l'émotion et définit son caractère altruiste. Le désir constitutif de l'affection est, selon Aristote, un désir rationnel (boulèsis). Le désir altruiste est le désir du bien d'autrui, lequel désir exige sa satisfaction. En d'autres termes, il est aussi désir de faire le bien de l'ami (1166b34), c'est-à-dire d'être un agent actif dans la production de son bien. Il est en outre un désir désintéressé qui ne saurait

${ }^{15}$ C'est le cas de Poséidon pour les Grecs (Il., 13.15-6; 15.44), d'Apollon pour le corps d'Hector (24.19-20), de Zeus pour les Grecs lors de la mort de Patrocle (19.340) pour Priam (24.332).

${ }_{16}$ À l'inverse. celui qui est sans compassion (nèleès) sur le champ de bataille blesse et tue d'un bronze sans pitié. Cf. J. Kiм (The Pity..., p. 66).

${ }^{17}$ C'est pourquoi certains définissent aujourd'hui l'émotion comme une force motivationnelle. 
faire toutefois de l'affection une émotion désintéressée dans la mesure où l'émotion en tant qu'attitude évaluative est par définition intéressée. Elle implique, en effet, le jugement : «ceci m'est utile ou nuisible». Il en est de même de la compassion qui ne saurait être purement désintéressée, puisqu'elle me renvoie à ma propre vulnérabilité. Intérêt et désintérêt ne sont pas mutuellement exclusifs et coexistent au contraire dans l'émotion. Le désintérêt s'exprime, comme l'affirme Aristote, par le fait de désirer le bien de l'ami pour l'ami lui-même et non pour en tirer un avantage ou du plaisir. Cela ne signifie pas que plaisir et utilité soient absents de l'affection, ils l'accompagnent au contraire, nous y reviendrons.

Nous allons montrer que d'une part, l'affection est nécessairement accompagnée de compassion dans la mesure où ces deux émotions ont pour constituant un même désir altruiste et d'autre part, que ces deux émotions sont au fondement du lien d'amitié. C'est en effet parce qu'il y a affection, qu'il y a amitié. L'affection est une condition et une composante de l'amitié ; elle en est aussi le symptôme. Il y a dans l'amitié un bénéfice mutuel bien que l'amitié ne soit pas en vue d'un tel bénéfice ${ }^{18}$. L'amitié suppose une affection réciproque ${ }^{19}$ qui implique une certaine inti-

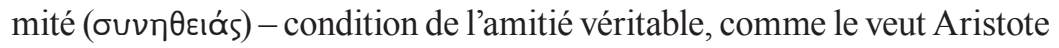
(1167a 12). Cela parce que l'intimité suppose de connaître et d'aimer le caractère de chacun (1157a 11-2). Il y a derrière l'intimité l'idée d'authenticité : parler de ses faiblesses à l'ami, ne pas feindre d'être ce qu'on n'est pas (Rhét. 1381b26-30). Fiabilité, loyauté et confiance sont des composantes essentielles de l'amitié. L'amitié, chez Homère comme chez Aristote, est un lien d'affection qui implique altruisme, réciprocité, engagement, intimité et reconnaissance mutuelle ${ }^{20}$.

C'est au regard de cette définition qu'il faut comprendre que le désir du bien de l'ami embrasse le désir de supprimer ou d'alléger ses souffrances. En d'autres termes, le désir constitutif de l'affection est inclusif du désir constitutif de la compassion. Cette relation inclusive est déter-

\footnotetext{
${ }^{18}$ M. Nussbaum, The Fragility of Goodness, Cambridge, Cambridge University Press, 2001, p. 355.

${ }^{19}$ Il est important de noter qu'une entorse à la réciprocité peut constituer un motif de rupture de l'amitié et l'extinction de l'affection.

${ }^{20}$ Cf. D. Konstan, Friendship in the Classical World, Cambridge, Cambridge University Press, 1997, p. 69. Je citerai ici E. HATFIELD \& R. L RAPSON qui distinguent entre passionnate love et compasionnate love qui « combines feeling of deep attachment, commitment and intimacy» (cité par Konstan, p. 177; E. HATFIELD and R. L RAPSON, 'Love and Attachment Process' in M. Lewis and J. M. Haviland-Jones, Handbook on Emotions, New York, Guilford Press, 2000, p. 654-62, p. 654-655).
} 
minante pour comprendre l'amitié. Le spectacle des souffrances de l'ami ne nous laisse jamais indifférent et produit des réactions émotionnelles variées dont la compassion est sans doute la plus manifeste. Ainsi les souffrances des Grecs produisent chez Patrocle la compassion et le désir conjoint de les alléger (Il.11.814). De même, les dieux qui éprouvent de la compassion pour les hommes agissent en vue de diminuer leurs souffrances : Apollon protège le corps d'Hector; Zeus envoie Hermès accompagné Priam à travers le camp grec comme il envoie Athéna nourrir de nectar et d'ambroisie un Achille affligé de douleur ; Circé, touchée par la compassion, se décide à accueillir avec hospitalité Ulysse et ses compagnons $(O d$. 10.339). Cette protection induite par la compassion justifie le fait que les hommes fassent appel à la compassion divine.

Cependant, selon Aristote, on ne peut pas éprouver de la compassion (éléos) pour l'ami parce que la relation est trop étroite et l'identification totale. Une trop grande proximité à l'autre constitue, selon lui, un obstacle. D. Konstan suggère que (1) l'ami est un autre soi-même, et (2) on ne ressent pas de pitié pour soi-même, d'où : on ne ressent pas de pitié pour l'ami. On a de la commisération pour l'ami, mais non de la pitié. Pourtant l'apitoiement sur soi est une expérience que chacun d'entre nous peut faire. Il s'agit ici d'une question de terminologie. Aristote reconnaît logiquement qu'on souffre avec l'ami de ses souffrances (il est un autre soi-même). Le terme utilisé est sunalgein, littéralement «souffrir avec», c'est-à-dire «compatir» (Rhét. 1385 a 5) $)^{21}$. Cependant, la compassion telle que nous la rencontrons chez Homère diffère de celle d'Aristote en ce qu'elle implique, nous l'avons vu, un rapport subtil de distance et de proximité que la définition aristotélicienne de l'amitié véritable ne saurait accepter puisque toute distance est annulée ${ }^{22}$. Une telle identification absolue de l'ami à soi est toutefois exceptionnelle et sans doute unique, comme le reconnaît lui-même Aristote (EN 1156b24-25). Cela n'est pas le cas de l'amitié entre Achille et Patrocle puisque bien qu'Achille considère Patrocle comme un autre soi-

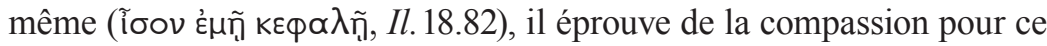
dernier. La forme d'identification induite par l'amitié n'exclut donc pas la compassion chez Homère, qui associe clairement compassion et affection : «Si tu éprouves pour lui de la compassion (oikteireis) s’il est cher à ton

\footnotetext{
${ }^{21}$ Nous rencontrons également les verbes sullupesthai et sunakhthesthai. Ces composés traduisent différentes sortes de souffrance : lupê (la peine, tristesse), akhthos (accablement, souci, chagrin), algô (douleur physique ou morale, agiter, troubler).

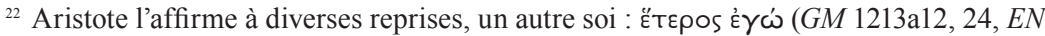
1169b7).
} 
cœur (philos)» (Il.23.548). Cela signifie que pour Homère l'identification se distingue de l'identité qui abolit toute distance.

Remarquons à cet égard que l'appel à la compassion suppose une tentative pour établir une relation d'amitié ${ }^{23}$. «Sois compatissant à mon égard et nous serons amis», semble dire Lycaon à Achille, lui rappelant ce qu'il a fait pour lui et qui constitue une marque d'amitié (le partage du pain, un accroissement de sa timè par une rançon : Il.21.74-96). Achille montre ironiquement qu'il a perçu la demande d'amitié de Lycaon en l'appelant «ami», mais il la rejette et le tue. Par ailleurs, c'est au nom de l'affection qui les unit qu'Andromaque appelle Hector à la compassion et l'enjoint de ne pas affronter Achille, c'est-à-dire aussi de la sauver, elle et son fils, de l'esclavage (Il.6.407, 6.431-2). C'est au nom de l'amitié (philophrosunè : Il. 9.256) qu'Ulysse demande à Achille de compatir aux maux des Grecs (qui ne sont pas responsables de son conflit avec Agamemnon, comme Ulysse semble le suggérer) et de retourner au combat (300-02), que Phénix (585) ainsi qu'Ajax (641-42) l'implorent. Un des arguments essentiels des ambassadeurs pour persuader Achille de retourner au combat est le lien d'amitié qui l'unit aux Grecs et qui implique la compassion et, par conséquent, le désir de sauver les Grecs de la catastrophe. Bien qu'Achille soit sensible à l'argument (645-46), l'émotion de colère l'emporte sur toute autre émotion et l'annule en quelque sorte, comme il le reconnaît («mon cœur se gonfle de colère» : 646).

Achille envahit par la colère est incapable de cette sympathie mutuelle, cette composante de l'affection nous l'avons vu, qui entre dans la définition de l'amitié. Par contre, il est immédiatement touché par les larmes de Patrocle rentrant du camp grec (Il. 16.5), manifestant un attendrissement pour l'ami, une sensibilité à sa souffrance qu'il cherche à comprendre (16.120). Ce désir de compréhension est aussi désir de comprendre comment soulager la souffrance de Patrocle. Mais les larmes de Patrocle qui soulèvent la compassion d'Achille sont elles-mêmes le produit de la compassion, celle de Patrocle pour les Grecs qui périssent sous les coups troyens. Le désir de protéger et d'aider les amis est présenté chez Homère comme inséparable de la compassion. C'est la compassion qui conduit Patrocle a retourné au combat et Achille à accepter que Patrocle revête ses armes.

Patrocle est dans l'Iliade une figure de l'être de compassion : attentif aux amis il a souci d'eux (19.287-300) $)^{24}$. Bien que le souci de l'autre ne

${ }^{23}$ Cf. M. Scott, Pity and Pathos in Homer, p. 11.

${ }^{24}$ Le narrateur nous le montre déchiré entre sa loyauté pour Achille et sa pitié pour les Grecs (11.814), qui finalement l'emportera, ayant été ébranlé par le discours persuasif de 
saurait se confondre avec la compassion - il s'en distingue essentiellement parce qu'il n'est pas une émotion - il en est un composant essentiel, comme il l'est de l'affection ${ }^{25}$. En effet, en compatissant aux souffrances de l'ami,

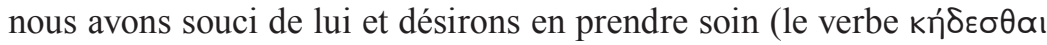
admet les deux sens). Le souci pour l'ami est exprimé à diverses reprises chez Homère. Citons la plainte d'Hécube après la mort d'Hector : «vivant, tu étais cher aux dieux, et ils ont encore souci de toi dans la mort fatale» (Il. 24.749-50) ou encore Achille présenté au chant I comme ayant souci des Grecs parce qu'ils sont ces philoi. La compassion est constituée du désir de prendre soin de l'autre, mais il est possible de prendre soin d'autrui sans éprouver de compassion; ce peut être par devoir. Mais l'aide et la protection qu'on assure à l'ami en détresse sont le résultat de la compassion qui fait que dans l'affection il n'y a, à strictement parler, ni devoir ni obligation ${ }^{26}$.

Il est intéressant de noter que si, comme nous l'avons montré, la compassion est constitutive de l'affection, la compassion peut produire l'affection. C'est le cas de Priam et d'Achille. La compassion a pour vertu de transformer l'hostilité entre les deux hommes en amitié. Si l'hostilité entre deux amis est impossible ${ }^{27}$, la compassion chasse l'hostilité (l'éris) et permet à l'amitié de s'imposer. Un lien d'amitié peut donc naître d'un geste de compassion, parce que lui-même produit la gratitude qui est un composant de l'amitié, comme le veut Aristote. La compassion débouche à l'occasion sur un acte qui provoque la gratitude ou permet le don généreux, la kharis. La kharis d'Achille à l'endroit de Priam est de lui accorder du temps pour les funérailles d'Hector. Priam exprime immédiatement sa gratitude à l'égard d'Achille : «tu m'accorderais une faveur» (Il. 24.661). Il s'agit de la part d'Achille d'un acte de désintérêt pur, c'est-à-dire de pure générosité. La kharis en tant que gratitude est une réaction émotionnelle à un acte altruiste dont nous sommes récipiendaires et qui suppose de la part du bienfaiteur un total désintérêt. La relation entre Achille et Priam illustre le caractère éminemment positif de la compassion. Nous consta-

Nestor et reprochant à Achille son manque de pitié (ónnvís : 16.35). Cf. l'analyse de O. TAPLIN (Homeric Soundings..., p. 175-178).

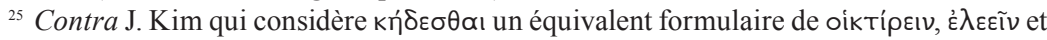

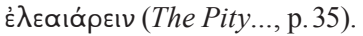

${ }^{26}$ Certains commentateurs ont noté que le lien de philotês auquel Ajax fait appel est un lien d'affection sur lequel est basée l'amitié, qui implique par conséquent que l'amitié chez Homère ne saurait être uniquement pensée en termes institutionnels et d'intérêts réciproques. Cf. J.T. Hooker, Homeric pí̉os, pp. 46 et ss. Contra E. Benveniste, Le vocabulaire..., vol. 1, p. 344.

${ }^{27}$ Aristote fait de l'amitié une médiété entre la flatterie et l'animosité. 
tons donc une relation particulièrement étroite entre compassion et amitié : l'amitié induit nécessairement la compassion et la compassion débouche à l'occasion sur l'amitié.

\section{Le plaisir comme apaisement (restauration) et joie}

Nous allons maintenant nous interroger sur le plaisir propre à l'amitié tel que nous le rencontrons chez Homère à partir des deux types de plaisir que distingue Aristote. Aristote classe les émotions en trois catégories : premièrement, celles accompagnées de peine, deuxièmement, celles accompagnées de plaisir et enfin les émotions mixtes (plaisir et peine). Le terme grec kata signifie ici que le plaisir et la peine entre dans la définition de l'émotion ${ }^{28}$, plutôt que quelque chose conçue séparément. Il n'est pas d'émotion sans peine ou plaisir et ce qui est cause de peine ou de plaisir est poursuivi ou évité (De Anima 431 a8-16). Certaines émotions peuvent donc être recherchées parce qu'elles procurent du plaisir, d'autres évitées parce qu'elles procurent de la peine ${ }^{29}$. Cependant si nous reconnaissons avec Aristote qu'il existe différents types de plaisir (EN 10.1175a22-28) et d'intensité de plaisir (1173 a 17), nous devons admettre que le plaisir et la peine sont spécifiques à chaque émotion. Le plaisir est la sensation d'une certaine émotion (Rhét. 1370a32). En tant qu'émotion, l'affection est accompagnée de plaisir tandis que la compassion est accompagnée de peine. Selon Aristote, l'amitié homérique n'est donc pas accompagnée d'un plaisir pur. Il n'est pas certain du reste que la compassion chez Homère soit une émotion dépourvue de tout plaisir, comme le veut Aristote, nous allons le voir.

Les usages d'hèdonè comme de lupè sont variables : des plaisirs du corps aux différentes attitudes mentales du simple plaisir à la joie avec toutes les variations possibles. Hèdonè possède, selon Cooper, la connotation de «positive mental excitement $»^{30}$. Pour Aristote, le véritable

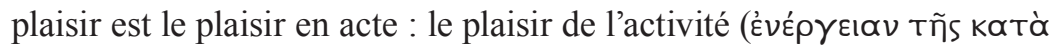

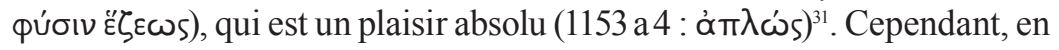
Rhétorique (1369b35-37), il définit le plaisir comme «un mouvement de l'âme et sa disposition soudaine et sensible dans un état naturel et la peine

${ }^{28}$ Cf. S. Leighton, 'Aristotle and the Emotions', in E. Rorty (ed.), Essays on Aristotle's Rhetoric, Berkeley, University Press of California, 1996, p. 218.

${ }^{29}$ L'émotion comme le plaisir et la peine sont donc du genre de l'assentiment et du refus (Rhét. 1378a).

${ }^{30}$ J. COOPER, 'An Aristotelian Theory of the Emotions', in E. RORTY (ed.), Essays on Aristotle's Rhetoric..., p. 246.

${ }^{31}$ Cf. EN 1152b33-1153a2. 


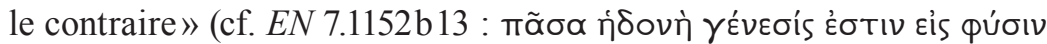

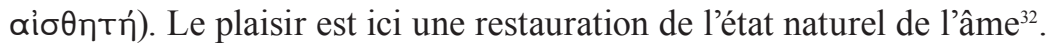
Le fait que le désir (orexis, boulèsis) soit constitutif de l'émotion justifie le fait que le plaisir soit compris comme satisfaction, c'est-à-dire comme un processus. L'émotion est, nous l'avons vu, une perturbation momentanée de la conduite dont le passage à l'action produit un retour à l'équilibre physiologique et mental. La restauration est fondamentale dans le processus émotionnel puisqu'elle le clôt. Combler un besoin ou un désir engendre le plaisir de la restauration, ou satisfaction, qui en tant que retour à l'équilibre correspond à la fin de la tension émotionnelle. Nous allons voir que l'amitié homérique induit ces deux types de plaisir de manière inclusive. Je m'appuierai donc sur la distinction aristotélicienne de ces deux formes de plaisir en définissant le premier comme relevant de l'apaisement et le second comme relevant du contentement, sans toutefois faire mienne la distinction entre processus et activité qui me semble inadéquate pour comprendre le plaisir et la peine qui accompagnent l'amitié homérique ${ }^{33}$.

Le terme lupè utilisé par Aristote couvre à la fois la douleur physique et toutes sortes de réponses et d'attitudes mentales négatives. Hors du vocabulaire philosophique, lupè signifie un sentiment assez fort : le chagrin, la tristesse, l'affliction. Selon J. Cooper, il faut entendre lupè dans son usage aristotélicien comme signifiant «distress», «feeling upset $\rangle^{34}$. La compassion est une émotion accompagnée de peine (chagrin, tristesse, affliction) parce que nous souffrons avec l'ami. La peine est, à cet égard, partie intégrante de la compassion. Toutefois, la souffrance de l'ami ne nous appartient pas : elle ne devient pas nôtre dans le processus émotionnel. C'est pourquoi la compassion n'est pas l'empathie, comme nous

${ }^{32}$ Cette définition du plaisir est empruntée au Philèbe (53c-55a). Aristote affirme que ce plaisir est un plaisir accidentel (EN 1152b34). Il précise au livre X de l'EN qu'il ne s'agit pas en fait d'une forme de plaisir en tant que tel, mais que nous ressentons du plaisir dans le processus de restauration de l'état naturel (1173b11-4). Pour un rejet du plaisir comme appartenant au genre du mouvement, par conséquent, comme processus cf. Top. 121 a31-40.

${ }^{33}$ Rappelons que la distinction entre les deux formes de plaisir recoupe chez Aristote celle

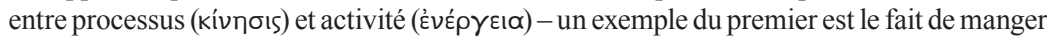
lorsqu'on a faim tandis que la seconde relève de la perception et de la pensée. Cette distinction n'est cependant pas toujours respectée par Aristote et est problématique dans bien des cas, comme l'ont noté un certain nombre de commentateurs. Cf. D. Bostock, Pleasure and Activity in Aristotle's Ethics, Phronesis 33, 1998, p. 251-272, D. FredE, 'Pleasure and Pain', in R. KRAUT (ed.), The Blackwell Guide to Aristotle's Nicomachean Ethics, Oxford, Blackwell, 2005, p. 263-271. Par exemple, qu'en est-il du plaisir de jouer au piano Voiles de Debussy, qui n'est pas, selon la définition aristotélicienne, une activité mais un processus ?

${ }^{34}$ J. COOPER, An Aristotelian Theory..., p. 246. 
l'avons indiqué plus haut. En d'autres termes, il existe une différence irréductible entre la souffrance qui est le propre de l'émotion et la souffrance de l'ami ${ }^{35}$. Achille souffre pour et avec Patrocle, mais sa souffrance en tant que telle lui reste extérieure. Cette altérité fondamentale de sa souffrance à la souffrance de Patrocle rend possible, nous l'avons déjà noté, la possibilité d'agir en vue de diminuer ou d'éliminer la souffrance de l'ami. L'arrachement à certaines souffrances selon leur degré exige une main qui ne peut se tendre que parce qu'elle est dans une position d'extériorité ${ }^{36}$.

Le caractère statique de la souffrance, qui est rivée au moment présent, s'oppose au caractère dynamique de la compassion qui voit au-delà, c'est-à-dire la fin de la souffrance. C'est justement cette fin que désire le compatissant et qu'il peut désirer du fait même qu'il ne subit pas ce que subit l'autre. Nous pourrions par commodité et pour maintenir l'altérité de ces deux souffrances recourir à une distinction terminologique quelque peu artificielle et utiliser le terme «douleur» pour dire la souffrance du sujet compatissant et le terme «souffrance» pour exprimer la souffrance de l'objet de la compassion.

La réalisation du désir de cessation de la souffrance chez autrui, c'està-dire l'action compassionnelle est source de plaisir en tant que plaisir de la restauration. C'est pourquoi la compassion est une émotion mixte qui consiste premièrement à éprouver de la douleur devant le spectacle d'une souffrance pour un mal immérité, deuxièmement, à ressentir le désir de diminuer ou de supprimer cette souffrance, qui appelle la réalisation de ce désir : l'action compassionnelle. Le processus émotionnel s'achève dans l'apaisement en tant qu'il est la satisfaction d'un désir et la fin de sa souffrance causée par la souffrance de l'ami. Le plaisir comme joie n'est cependant pas absent de la compassion. Il s'agit d'une part, d'une forme de contentement de soi puisque la compassion suscite le plaisir de se sentir moralement bon : on éprouve en effet une émotion socialement positive et on donne une réponse effective à la souffrance d'autrui. D'autre part, la réponse effective qu'est l'action compassionnelle en tant qu'action bonne ou vertueuse est source de joie. L'activité vertueuse est, selon Aristote, source de plaisir (EN 1173b30). Remarquons que ce n'est pas, à propre-

\footnotetext{
${ }^{35}$ «Admettons que moi, par exemple je puisse souffrir profondément, un autre ne pourra jamais connaître l'étendue de ma souffrance parce qu'il est un autre que moi», dit Yvan à Aliocha (DostoḮvski, Les frères Karamazov, trad. franç. Paris, Livre de Poche, p. 300).

${ }^{36}$ « Il y a dans la souffrance, écrit E. Lévinas, une absence de tout refuge. Elle est le fait d'être directement exposé à l'être. Elle est faite de l'impossibilité de fuir et de reculer. Toute l'acuité de la souffrance est dans cette impossibilité de recul» (E. LÉVINAs, Le temps et l'autre, Paris, PUF, Quadrige, 1985, p. 55.).
} 
ment parler, l'émotion qui engendre le contentement, elle ne l'engendre qu'en tant qu'elle engendre l'action compassionnelle.

Il nous faut à présent examiner s'il existe un plaisir spécifique lié à l'attitude compassionnelle à l'égard de l'ami dont la souffrance soulève une émotion complexe faite d'affection, de compassion et de peur. Remarquons d'abord que le plaisir de se sentir moralement bon disparait dans le cas de l'ami puisque la compassion dans le cas de l'amitié est le fruit exclusif de l'affection. On n'éprouve pas de plaisir à se sentir moralement bon à secourir l'ami. Bien qu'il y ait un plaisir qui accompagne l'action vertueuse, qu'elle soit ou non dirigée vers l'ami, ce plaisir est accidentel ou par surcroît parce que, à proprement parler, l'affection est moralement neutre. Dans le cas de l'amitié et à la différence de la compassion c'est la nature plaisante de l'action compassionnelle à laquelle est associée l'émotion (l'action compassionnelle pour l'ami) qui rend la restauration plaisante et qui en fait une restauration plaisante par accident. Cependant, bien que la réalisation du désir compassionnel dans l'amitié soit un apaisement, l'action compassionnelle est plus que la résolution de la tension produite par la compassion puisqu'il y entre de l'affection ${ }^{37}$.

Le degré et la forme d'engagement, c'est-à-dire la nature de l'action compassionnelle, et le degré de la douleur ressentie varient, en effet, en fonction de l'objet : ami ou inconnu. L'affection guide la nature, l'engagement et l'intensité de l'action compassionnelle. On peut en outre remarquer que lorsqu'il y a affection, l'intensité de l'émotion et corrélativement le degré d'engagement dans la réponse donnée sont proportionnels au degré d'affection - la compassion d'Achille pour Priam est moins intense que celle d'Achille pour Pélée ou Patrocle ${ }^{38}$. En conséquence, le plaisir lié à la réponse émotionnelle est plus intense et corrélativement la douleur. L'urgence de la réponse à donner est plus grande dans le cas de l'amitié. L'affection est, en ce sens, première par rapport à la compassion. En outre, l'action compassionnelle dans l'amitié n'est pas accomplie en vue de l'apaisement, mais parce que le lien d'affection la requiert. Il en est ainsi parce que la satisfaction liée à la résolution de la tension émotionnelle (la cessation de la douleur) est secondaire. La cessation suit l'action compassionnelle pour l'ami, mais elle n'est pas visée de manière immédiate et première comme

\footnotetext{
${ }^{37}$ Il ne me semble pas possible ici en suivant la suggestion de D. Bostock d'affirmer qu'à strictement parler une telle satisfaction est un plaisir absolu, c'est-à-dire contentement et non apaisement, dans la mesure où il y a plaisir parce qu'il y a perception, conscience de la satisfaction - qui est une activité (Pleasure and Activity..., p. 269). Un plaisir sans conscience n'est pas un plaisir.

${ }^{38}$ L'intensité de l'affection est indiquée par le superlatif philtatoi (Il. 9.586).
} 
dans le cas de l'action compassionnelle où n'entre pas l'affection. La cessation de la peine est par surcroît, ou accidentelle, comme l'est par conséquent le plaisir en tant qu'apaisement. En outre, si comme le veut Aristote, le plaisir absolu, c'est-à-dire le contentement, est constitutif de l'affection (EN 1156b24-26), alors le contentement est premier par rapport à l'apaise-

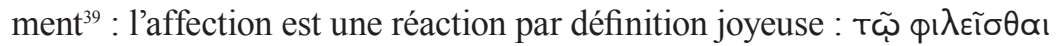

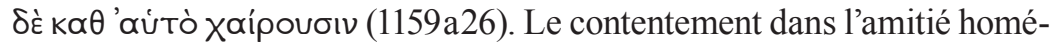
rique est donc premier par rapport au plaisir de la restauration produit par la résolution de la tension émotionnelle liée à la compassion.

Nous pourrions ranger le plaisir de l'amitié dans la catégorie aristotélicienne des plaisirs bons à rechercher. D'une part, c'est un plaisir noble puisque sa source est elle-même noble (cf. EN 1173b29-35) et d'autre part, c'est un plaisir désirable en lui-même. Selon Aristote, le plaisir en tant que contentement renforce notre excellence dans l'activité (EN 1153a23-4, 1174a31-1175b 1). Par conséquent, le plaisir constitutif de l'amitié renforce également notre excellence dans l'amitié, c'est-à-dire notre capacité à désirer et à faire le bien de l'ami. En outre, la perfection de l'activité d'où résulte le plaisir implique la perfection du plaisir. En d'autres termes, un plaisir parfait est constitutif d'une activité parfaite ${ }^{40}$, mais en même temps le plaisir parfait l'activité (EN 1174b20-4) : il s'agit d'un surcroît de perfection

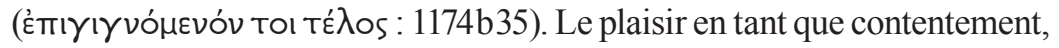
est, en un sens, le symptôme de l'activité parfaite.

Le plaisir propre à l'amitié est une forme de contentement qui dépend à la fois des circonstances extérieures, de l'ami, de son attitude envers nous et de notre attitude envers lui. S'il est agréable de passer du temps avec l'ami (Rhét. 1381a31), le bonheur de l'ami participe de notre propre bonheur : «l'ami se réjouit avec nous de notre bien», dit Aristote. C'est pourquoi les amis sont une source mutuelle de plaisir (EN 1157b37), mais un plaisir qui n'est pas, comme le veut Aristote, sans mélange puisque le plaisir de l'amitié homérique n'est pas exempt de douleur, l'amitié n'étant pas sans la compassion.

Je conclurai en montrant que le plaisir de l'amitié est un plaisir vertueux. Nous avons vu que bien que les deux formes de plaisir accompagnent l'amitié homérique, le plaisir de la restauration, c'est-à-dire l'apaisement, est secondaire par rapport à l'action que cette dernière engendre. Ce

\footnotetext{
${ }^{39}$ Le plaisir de l'amitié (relation) n'est pas ici différent du plaisir de l'affection - bien que l'affection ne soit pas une activité (exemple où la distinction activité et processus n'est pas respectée).

${ }^{40}$ Plaisir et activité sont à ce point inséparables qu'ils semblent se confondre (EN 1175b34-35).
} 
point est important parce que la dimension égoïste de la compassion telle que définie et qui pourrait être une objection à sa qualité vertueuse disparaît dans l'amitié homérique. La compassion parce qu'elle est une émotion pénible qui porte en elle le désir de supprimer la douleur ressentie du fait de la souffrance de l'autre est égoïste. L'action compassionnelle est motivée par le désir de supprimer la souffrance de l'autre parce que ce faisant elle supprime notre propre douleur. L'action compassionnelle est, en effet, le fruit d'une double motivation : altruiste et égoïste. Mais dans l'amitié il en va différemment puisque la satisfaction liée à la résolution de la tension émotionnelle (la cessation de la douleur) est secondaire, nous l'avons vu. Il n'en reste pas moins un aspect égoïste irréductible dans l'amitié ${ }^{41}$.

L'amitié est un lien essentiel dans le domaine pratique dans la mesure où (1) elle mobilise des émotions positives et ne soulève aucune émotion négative ; (2) elle constitue un obstacle à celle-ci ; (3) elle nous conduit à désirer le bien de l'ami non pour notre intérêt uniquement, mais pour le sien propre (le bien de l'ami est aussi notre bien ; «dans l'amitié tout est commun », rappelle Aristote (EN 1159b32) ; (4) elle induit un comportement nécessairement vertueux. L'amitié a donc une valeur saillante sur le plan éthique. En cultivant l'amitié, nous cultivons des émotions socialement et éthiquement positives et, en conséquence, des comportements vertueux et repoussons des émotions socialement négatives comme la jalousie, la haine, l'envie, le ressentiment, l'hostilité et l'injustice.

Le caractère socialement positif de l'amitié a été souligné par Aristote qui voit dans l'amitié un lien social puissant (EN 7.9), facteur de rapprochement des êtres humains et qui soude la communauté des hommes

${ }^{41}$ Aristote, on le sait, distingue trois types d'amitié et il n'est pas toujours aisé de voir si l'amitié dans le fond n'exprime pas tout type de lien social fondé soit sur le plaisir, l'utilité, la vertu ou les trois ensemble. Notons que l'amitié politique ou civique est fondamentale pour Aristote. Nous devons potentiellement établir une amitié selon la vertu avec nos concitoyens. Rappelons que si l'amitié véritable était possible à grande échelle, la justice ne serait pas utile ( $E N 1155$ a 25). L'amitié homérique embrasse les trois sortes d'amitiés aristotéliciennes puisque dans chacune d'entre elles, on agit en vue du bien de l'ami et désire son bien. Ces différents types d'amitié peuvent être compris comme différentes types de déploiement d'un même phénomène qui consiste à viser le bien mutuel dans des circonstances et situations différentes (cf. J. COOPER, 'Aristotle on Friendship', Knowledge, nature, and the good: essays on ancient philosophy, Princeton, Princeton University Press, 2004 p. 302). Ces types d'amitié peuvent être distingués en fonction de leur degré d'altruisme puisque le plaisir a incontestablement une dimension égoïste comme l'utilité. Il semble ici qu'il faille adopter une voie médiane et penser ces deux types comme l'affirme J. Cooper comme : « a complex and subtle mixture of self-seeking and unself-interested well-wishing and well-doing» (ibid., p.305). L'amitié selon la vertu inclut les deux autres types d'amitié puisque les deux amis prennent plaisir dans la compagnie de chacun et bénéficie de l'amitié de chacun. 
(EN 1155a23-25) - ce que nous voyons également à l'œuvre dans la scène finale de réconciliation entre Priam et Achille. La dimension éthique et sociale de l'amitié résulte également du fait que la compassion en est une composante essentielle. Chez Homère en effet la compassion contrecarre la sauvagerie - celui qui est sans compassion est tel un animal sauvage, ainsi Achille mutilant le corps d'Hector - et débouche sur l'hospitalité $(O d .17 .367)$. C'est ce qui fait de l'amitié chez Homère comme chez Aristote qu'elle s'accompagne de nombreuses vertus ou qu'elle est une sorte de vertu.

L'amitié est en outre, selon Aristote, une condition essentielle du bonheur. Elle en est du reste constitutive : il n'est pas de bonheur sans amitié (EN 1169 b 20-21). Dans l'amitié homérique, nous désirons le bonheur de l'ami et nous y participons activement par différents moyens renforçant ainsi notre comportement vertueux. Pour Aristote comme pour Homère, l'amitié est une attitude et une disposition vertueuses, qui engendre un comportement vertueux, source de plaisir.

\section{Título. Prazer e amizade: Homero e Aristóteles em diálogo.}

Resumo. O que a afeição e a compaixão têm em comum é o fato de serem emoções sociais, porém, de acordo com Aristóteles, se a primeira vem acompanhada de prazer, a segunda vem acompanhada de dor. Entretanto, segundo Homero (em contraste com Aristóteles), é possível sentir compaixão por um amigo. Questionamos, aqui, o vínculo entre compaixão e afeição e mostramos que, tomadas como emoções, elas consistem em um desejo de agir em prol do bem alheio, isto é, consistem em um desejo altruísta em Homero. A satisfação desse desejo produz um duplo prazer, manifesto sob a forma de contentamento e apaziguamento. Ilustramos nossa discussão, estabelecendo um diálogo entre Homero e Aristóteles.

Palavras-chave. Amizade; afeição; compaixão; prazer; apaziguamento; contentamento; emoção; intimidade; obrigação; desejo; altruísmo; vulnerabilidade; Aristóteles; Homero. 